\title{
Nonequilibrium dynamical ferromagnetism of interacting Single-Molecule Magnets
}

\author{
Gui-Bin Liu and Bang-Gui Liu* \\ Institute of Physics, Chinese Academy of Sciences, Beijing 100190, China \\ Beijing National Laboratory for Condensed Matter Physics, Beijing 100190, China
}

(Dated: October 31, 2018)

\begin{abstract}
We propose a nonequilibrium Monte Carlo (MC) approach to explore nonequilibrium dynamical ferromagnetism of interacting single molecule magnets (SMMs). Both quantum spin tunneling and thermally activated spin reversal are successfully implemented in the same MC simulation framework. Applied to a typical example, this simulation method satisfactorily reproduces experimental magnetization curves with experimental parameters. Our results show that both quantum and classical effects are essential to determine the hysteresis behaviors. This method is effective and reliable to gain deep insights into SMMs.

PACS numbers: 75.75.+a, 05.10.-a, 75.10.-b, 75.70.Ak, 75.90.+w
\end{abstract}

Introduction. The single-molecule magnet (SMM) is very interesting because of its potential applications in spintronic devices [1, 2], quantum computing [3], information storage [4], optical transistors 5], and so on. The most famous examples are $\mathrm{Mn}_{12}[6,7], \mathrm{Fe}_{8}[8]$, and $\mathrm{Mn}_{4}[$ [9] A SMM usually has well-defined spin ground state and large magnetic anisotropy, and can exhibit hysteresis loops at low enough temperatures [10]. LandauZener [11, 12] (LZ) model has been used to explain the steps of the hysteresis loops [10, 13, 14]. On theoretical side, numerically exact method has been used to the phenomenological spin model for the $\mathrm{Fe}_{8}$ and $\mathrm{Mn}_{12}$ systems, and calculated results show that LZ model is reliable for usual experimental field sweeping rates in describing the two-level problems at the level crossing fields [15, 16], but experimental hysteresis loops cannot be explained because thermally activated processes are not considered [10]. Thermally activated processes, however, play important roles in determining the nonequilibrium magnetization dynamics and even level populations. A quantitative method which is able to reproduce the full hysteretic magnetization dynamics of SMMs is in need.

Here, we propose a nonequilibrium Monte Carlo (NQMC) approach taking both the LZ quantum tunneling effect and the thermally activated processes into account, and hereby satisfactorily reproduce and explain experimental magnetization behaviors of SMMs.

Spin model. For simplicity without losing physics, we arrange SMMs on a finite two-dimensional (2D) rectangular lattice of $L_{1} \times L_{2}$, and consider inter-SMM magnetic dipolar and exchange interactions. Actually, some single layers of patterned SMMs has already been grown on specific solid surfaces [17, 18, 19], and it has been proved that a SMM, when put on a Au surface, can still keep its essential properties [4]. We use giant spin approximation. Every SMM has a spin of $S$. We use a typical lattice constant $a=1.5 \mathrm{~nm}$, which is an intermediate value of well-known SMMs. The model Hamiltonian reads

$$
H=\sum_{i} H_{i}^{0}+\frac{1}{2} \sum_{i \neq j}\left(J_{i j}^{\mathrm{di}}-J_{i j}\right) \mathbf{S}_{i} \cdot \mathbf{S}_{j},
$$

where $J_{i j}^{\text {di }}$ and $J_{i j}$ describe the magnetic dipolar and exchange interactions between spins at $i$ and $j$. The factor $1 / 2$ is due to the double counting in the summation. The first term describes the part for all the single SMMs, and $H_{i}^{0}$ is given by

$$
H_{i}^{0}=-D_{2}\left(\hat{S}_{i}^{z}\right)^{2}-D_{4}\left(\hat{S}_{i}^{z}\right)^{4}+H_{i}^{\mathrm{tr}}+g \mu_{B} B_{z} \hat{S}_{i}^{z},
$$

where $D_{2}$ and $D_{4}$ are positive anisotropic parameters, $g$ the Landé $\mathrm{g}$-factor (here $g=2$ is used), $\mu_{B}$ the Bohr magneton, $B_{z}(t)$ the external magnetic field in the $z$ direction. $\hat{S}_{i}=\left(\hat{S}_{i}^{x}, \hat{S}_{i}^{y}, \hat{S}_{i}^{z}\right)$ is the quantum spin operator for the $i$ th SMM. As for the transverse term $H_{i}^{\text {tr }}$, it is usually defined as $H_{i}^{\operatorname{tr}}=E\left[\left(\hat{S}_{i}^{x}\right)^{2}-\left(\hat{S}_{i}^{y}\right)^{2}\right]+g \mu_{B} B_{x} \hat{S}_{i}^{x}$, where $E$ is the second-order transverse anisotropic constant and $B_{x}$ is the transverse external field in the $x$ direction. As usual, $B_{x}$ is a constant and $B_{z}(t)$ is a linear function of the time $t$ with the sweeping rate $\nu$.

LZ tunneling probability. The easy axis determines two equilibrium spin directions in the $\pm z$ direction. When calculating the LZ tunneling probability, we treat the SMM spins coupled with the $i$ th spin by using the mean field approximation. As a result, for the $i$ th SMM, we have the effective one-SMM Hamiltonian

$$
H_{i}=H_{i}^{0}+g \mu_{B} B_{i}^{\text {eff }} \hat{S}_{i}^{z}
$$

where the effective mean field $B_{i}^{\text {eff }}$ is given by

$$
B_{i}^{\mathrm{eff}}=\sum_{j(\neq i)}\left(J_{i j}^{\mathrm{di}}-J_{i j}\right) S_{j}^{\mathrm{eq}} /\left(g \mu_{B}\right),
$$

where $S_{j}^{\text {eq }}$ is the equilibrium value $(S$ or $-S)$ for the $i$ th spin along the easy axis. This approximation is natural in the NQMC simulation, as will be clarified in the following. Then the standard diagonalization technique can be used to solve Eq. (3). Hamiltonian Eq. (3) has $2 S+1$ energy levels, which can be labeled by the quantum numbers $m=S, S-1, \cdots,-(S-1),-S$ to a first-order approximation. If without the transverse part $H_{i}^{\text {tr }}$, Eq. (3) is diagonal, and there are level crossings at some special field values. When the transverse term $H_{i}^{\text {tr }}$ is taken into 
account, the level crossings become avoided [10, 13, 14]. When $B_{z}$ is swept close to $B_{m, m^{\prime}}$ at which the avoided level crossing happens between states $m$ and $m^{\prime}$, quantum tunneling occurs between the two states. Strictly speaking, this tunneling is beyond LZ model which is a two-states theory [11, 12], but LZ tunneling can be used as a good approximation for each of the tunneling processes because LZ transition time is usually very short compared to time increments between the two successive avoided level crossings [15, 16, 20]. The nonadiabatic LZ tunneling probability $P_{m, m^{\prime}}$ is given by 11,12 ]

$$
P_{m, m^{\prime}}=1-\exp \left[-\frac{\pi \Delta_{m, m^{\prime}}^{2}}{2 \hbar g \mu_{B}\left|m-m^{\prime}\right| \nu}\right],
$$

where the tunnel splitting $\Delta_{m, m^{\prime}}$ describes the energy gap at the avoided crossing of states $m$ and $m^{\prime}$. $B_{m, m^{\prime}}$ and $\Delta_{m, m^{\prime}}$ can be calculated by diagonalizing Eq. (3).

Thermally activated reversal rate. In order to calculate thermal spin reversal rate, we use classical spin approximation which is reasonable and reliable because the spin usually is very large for SMMs. As a result, the energy of the $i$ th SMM is given by

$$
E_{i}=-D_{2}\left(S_{i}^{z}\right)^{2}-D_{4}\left(S_{i}^{z}\right)^{4}+h_{i} S_{i}^{z}
$$

where $h_{i}=g \mu_{B}\left(B_{z}+B_{i}^{\text {eff }}\right)$. Each of the spins has two equilibrium orientations $( \pm S)$ along the easy axis. We use the angle $\theta_{i}$ to describe the $i$ th spin's deviation from its original equilibrium orientation. All the other angle values $\left(0<\theta_{i}<\pi\right)$ are the transition states for the spin to reverse its orientation. We express $S_{i}^{z}$ as $S_{i}^{\text {eq }} \cos \theta_{i}$. Usually, there exists a maximum in the curve of $E_{i}\left(\cos \theta_{i}\right)$, and the maximum determines the energy barrier for the spin reversal [21, 22]. Defining $x_{i}=\cos \theta_{i}$, we have $-1 \leq x_{i} \leq 1$. Then the barrier is given by

$$
\Delta E_{i}= \begin{cases}E_{i}\left(x_{i}=x_{i}^{m}\right) & , \text { if }\left|x_{i}^{m}\right| \leq 1 \\ E_{i}\left(x_{i}=-1\right)=2\left|h_{i} S_{i}^{\mathrm{eq}}\right| & , \text { if } x_{i}^{m}<-1 \\ E_{i}\left(x_{i}=1\right)=0 & , \text { if } x_{i}^{m}>1\end{cases}
$$

where $x_{i}^{m}$ (according to the maximum) is defined as

$$
x_{i}^{m}=\sqrt[3]{-\frac{q}{2}+\sqrt{\Delta_{d}}}+\sqrt[3]{-\frac{q}{2}-\sqrt{\Delta_{d}}},
$$

where $\Delta_{d}=(q / 2)^{2}+(p / 3)^{3}, p=D_{2} /\left(2 D_{4} S^{2}\right)$, and $q=-h_{i} S_{i}^{\mathrm{eq}} /\left(4 D_{4} S^{4}\right)$. Then the reversal rate of the $i$ th spin is determined by the Arrhenius law [23], $R_{i}=$ $R_{0} \exp \left(-\Delta E_{i} /\left(k_{B} T\right)\right)$, where $k_{B}$ is the Boltzmann constant and $R_{0}$ the characteristic attempt frequency.

Simulation method and parameters. To set up our NQMC simulation, we define MC steps by the time points, $t_{n}=\Delta t \cdot n$, where $n$ nonnegative integers. Every spin takes either $S$ or $-S$ at each of the times $t_{n}$. A spin can be reversed by overcoming the thermal barrier and tunneling through LZ mechanism. The probability $P_{i}$ for

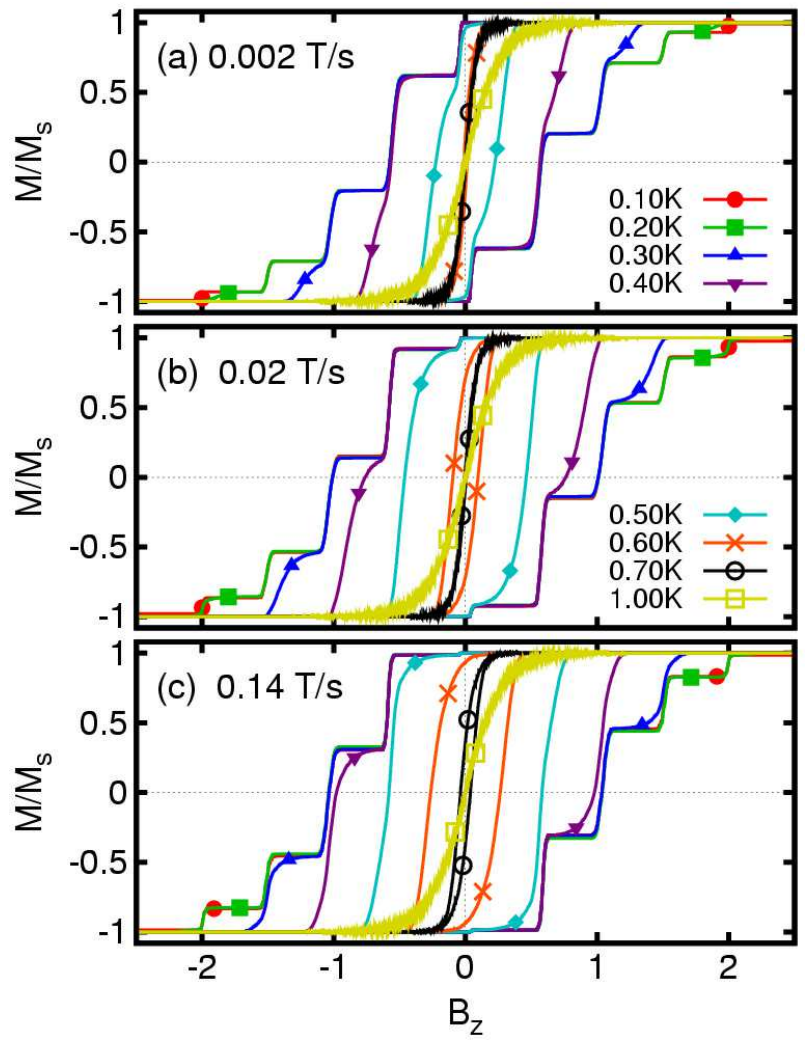

FIG. 1: (color online). Simulated magnetization curves $\left(M / M_{s}\right.$ vs. $B_{z}$ in $\left.\mathrm{T}\right)$ with three sweeping rates: $\nu=0.002$ (a), 0.02 (b), and 0.14 (c) T/s. For all the three panels, the temperatures are $T=0.10(\bullet), 0.20(\boldsymbol{\square}), 0.30(\mathbf{\Delta}), 0.40(\boldsymbol{\nabla})$, $0.50(\diamond), 0.60(\times), 0.70(\mathrm{O})$, and $1.00(\square) \mathrm{K}$.

a thermally activated spin reversal within a MC step is defined by $P_{i}=\Delta t \cdot R_{i}[21,22]$, and the LZ tunneling probability is given by Eq. (5). Actually, we have the magnetization $m=S$ at the beginning of field sweeping, and then need to consider only the avoided crossings at $\left(S, m^{\prime}\right)$, where $m^{\prime}=-S,-S+1, \cdots$. When the field is swept to $B_{S, m^{\prime}}$, we consider the LZ tunneling by adding the LZ probability $P_{S, m^{\prime}}$ to the thermally activated reversal probability. Because our Monte Carlo time step is much larger than the LZ transition and spin relaxation times, a spin will have long enough time to transit to the possible lowest level at current field after a LZ tunneling is finished 24]. Therefore, our NQMC simulation is self-consistent.

For convenience in comparing with experiment, we choose the $\mathrm{Mn}_{4}$ system to demonstrate the NQMC method and its power. Hence we have $S=9 / 2, D_{2} / k_{B}=$ $0.608 \mathrm{~K}, D_{4} / k_{B}=3.8 \mathrm{mK}$, and $E / k_{B}=32 \mathrm{mK}$ from Ref. 14. We assume that our $2 \mathrm{D}$ lattice is put on a metal surface and hence use a carrier-mediated 2D interaction for $J_{i j}\left(J / k_{B}=5.8 \mathrm{mK}\right.$ for the nearest spins) [25]. This inter-SMM interaction, weak enough, does not destroy the essential SMM properties. In addition, we already 
show that simulated results are not sensitive to specific choices of the interaction, and other interactions yield similar results. We take $B_{x}=0.02 \mathrm{~T}, \Delta t=1 \mathrm{~ms}$, and $R_{0}=10^{9} / \mathrm{s}$. Such parameter choices guarantee the good balance between computational demand and precision. In our simulations, field $B_{z}$ is swept from $-2.5 \mathrm{~T}$ to $2.5 \mathrm{~T}$ in the forward process, and the full hysteresis loop is obtained simply by using the loop symmetry. Every hysteresis loop is calculated by averaging over 100 runs to reduce statistical errors.

Simulated results and discussions. We have done our NQMC simulations with various $\left(L_{1}, L_{2}\right)$ values and experimentally accessible sweeping rates $\nu(0.002 \sim 0.14$ $\mathrm{T} / \mathrm{s})$ and temperatures $T(0.10 \sim 1.00 \mathrm{~K})[14]$. Presented in Fig. 1 are our simulated magnetization $M$ (normalized to the saturated value $M_{s}$ ) curves against the applied sweeping field $B_{z}$ with $L_{1}=L_{2}=10$ for three sweeping rates, namely $0.002,0.02$, and $0.14 \mathrm{~T} / \mathrm{s}$. For each of the three cases, we present the results for eight temperatures: $0.10,0.20,0.30,0.40,0.50,0.60,0.70$, and $1.00 \mathrm{~K}$. It is clear that there exist hysteresis loops for low enough temperatures and complete paramagnetic magnetizations are obtained for high enough temperatures. We can clearly see four steps between 0 and $2 \mathrm{~T}$ for low enough temperatures. In addition, we have done NQMC simulations for various values of $L_{1}$ and $L_{2}$. For square lattices $\left(L=L_{1}=L_{2}\right)$, the step heights decrease a little with increasing $L$ from 8 to 50 . For rectangular lattices with $L_{1} \times L_{2}=24^{2}$, the step heights change a little for different $L_{1}$ and $L_{2}$. It is clear that the step structures are far from those predicted by LZ tunneling alone.

In fact, thermally activated reversals play important roles in determining the magnetization curves. Our simulations show that the number of the steps decreases with increasing the temperature for given value of the sweeping rate $\nu$. It can be seen that the area enclosed by a hysteresis loop increases monotonically with increasing the sweeping rate and decreasing the temperature, and the smallest visible loop is at $0.70 \mathrm{~K}$ for $\nu=0.14 \mathrm{~T} / \mathrm{s}$, $0.60 \mathrm{~K}$ for $0.02 \mathrm{~T} / \mathrm{s}$, and $0.50 \mathrm{~K}$ for $0.002 \mathrm{~T} / \mathrm{s}$, respectively. The thermal effect increases substantially when we increase the temperature from $0.3 \mathrm{~K}$ on. There will be no step structures for usual sweeping rates when the temperature is higher than $0.5 \mathrm{~K}$. The hysteresis loop will disappear when the temperature is too high.

Conclusion. We have proposed a NQMC approach to explore the nonequilibrium dynamical ferromagnetism of interacting SMMs. Both quantum spin tunneling and thermally activated spin reversal are successfully implemented in the same Monte Carlo simulation framework. Applied to the $\mathrm{Mn}_{4}$ system, this simulation method satisfactorily reproduces experimental magnetization curves with experimental parameters. Our results show that both quantum and classical effects are essential to determine the hysteresis loops in the magnetization curves. This method is effective and reliable, and can be used to gain deep insights into SMMs and other quantum nanomagnets with strong anisotropy.

This work is supported by Nature Science Foundation of China (Grant Nos. 10874232 and 10774180), Chinese Department of Science and Technology (Grant No. 2005CB623602), and Chinese Academy of Sciences (Grant No. KJCX2.YW.W09-5).

* Corresponding author: bgliu@aphy.iphy.ac.cn

[1] A. R. Rocha, V. M. García-suárez, S. W. Bailey, C. J. Lambert, J. Ferrer, and S. Sanvito, Nature Mater. 4, 335 (2005).

[2] L. Bogani and W. Wernsdorfer, Nature Mater. 7, 179 (2008).

[3] M. N. Leuenberger and D. Loss, Nature 410, 789 (2001).

[4] M. Mannini, F. Pineider, P. Sainctavit, C. Danieli, E. Otero, C. Sciancalepore, A. M. Talarico, M.-A. Arrio, A. Cornia, D. Gatteschi, and R. Sessoli, Nature Mater. 8, 194 (2009).

[5] J. Hwang, M. Pototschnig, R. Lettow1, G. Zumofen, A. Renn, S. Götzinger1, and V. Sandoghdar, Nature 460, 76 (2009).

[6] T. Lis, Acta Crystallogr., Sect. B 36, 2042 (1980).

[7] R. Sessoli, D. Gatteschi, A. Caneschi, and M. A. Novak, Nature 365, 141 (1993).

[8] K. Weighardt, K. Pohl, I. Jibril, and G. Huttner, Angew. Chem. Int. Ed. 23, 77 (1984).

[9] J. S. Bashkin, H. R. Chang, W. E. Streib, J. C. Huffman, D. N. Hendrickson, and G. Christou, J. Am. Chem. Soc. 109, 6502 (1987).

[10] D. Gatteschi, R. Sessoli, and J. Villain, Molecular nanomagnets, Oxford University Press, New York, 2006.

[11] L. Landau, Phys. Z. Sowjetunion 2, 46 (1932).

[12] C. Zener, Proc. R. Soc. London, Ser. A 137, 696 (1932).

[13] W. Wernsdorfer, R. Sessoli, A. Caneschi, D. Gatteschi, A. Cornia, and D. Mailly, J. Appl. Phys. 87, 5481 (2000).

[14] W. Wernsdorfer, S. Bhaduri, A. Vinslava, and G. Christou, Phys. Rev. B 72, 214429 (2005).

[15] E. Rastelli and A. Tassi, Phys. Rev. B 64, 064410 (2001).

[16] P. Földi, M. G. Benedict, J. M. Pereira, Jr., and F. M. Peeters, Phys. Rev. B 75, 104430 (2007).

[17] G. G. Condorelli, A. Motta, I. L. Fragal, F. Giannazzo, V. Raineri, A. Caneschi, and D. Gatteschi, Angew. Chem. Int. Ed. 43, 4081 (2004).

[18] A. Naitabdi, J.-P. Bucher, P. Gerbier, P. Rabu, and M. Drillon, Adv. Mater. 17, 1612 (2005).

[19] J. Gómez-Segura, J. Veciana, and D. Ruiz-Molina, Chem. Commun. 2007, 3699 (2007).

[20] N. V. Vitanov, Phys. Rev. A 59, 988 (1999).

[21] Y. Li and B.-G. Liu, Phys. Rev. Lett. 96, 217201 (2006); Phys. Rev. B 73, 174418 (2006).

[22] B.-G. Liu, K.-C. Zhang, and Y. Li, Front. Phys. China 2, 424 (2007).

[23] S. Arrhenius, Z. Phys. Chem. 4, 226 (1889).

[24] K. Saito, S. Miyashita, and H. De Raedt, Phys. Rev. B 60, 14553 (1999).

[25] M. T. Béal-Monod, Phys. Rev. B 36, 8835 (1987); D. N. Aristov, Phys. Rev. B 55, 8064 (1997). 